Rosario Camacho Martínez

SECRETARIO

Juan Antonio Sánchez López

CONSEJO DE REDACCIÓN

Natalia Bravo Ruiz

Eugenio Carmona Mato

Isidoro Coloma Martín

Reyes Escalera Pérez

Francisco J. García Gómez

Ma de la O Heredia González

$M^{a}$ Teresa Méndez Baiges

Aurora Miró Domínguez

Juan Ma Montijano García

José Miguel Morales Folguera

F. Javier Ordóñez Vergara

Francisco J. Palomo Díaz

Eva $M^{a}$ Ramos Frendo

Francisco J. Rodríguez Marín

Nuria Rodríguez Ortega

Belén Ruiz Garrido

Rafael Sánchez-Lafuente

María Teresa Sauret Guerrero

DISEÑO Y MAQUETACIÓN

Sonia Ríos Moyano

VINEETA DE LA PORTADA

Alonso Cano: Proyecto de

Tabernáculo (h. 1665).

Biblioteca Nacional (Madrid)

Homenaje a Alonso Cano en el

IV Centenario de su nacimiento

(1601-2001)

Esta revista es analizada por el centro de Información y Documentación Científica del C.S.I.C. e incluídas en la B.D.I.S.O.C.

EDITA: Departamento de Historia del Arte de la Facultad de Filosofía y Letras Servicio de Publicaciones de la Universidad de Málaga

Impreso en Andalucía

I.S.S.N.: 0211-8483

Depósito Legal: MA-1.554-2001 
-: Boletín de Arte, $n^{\circ} 22-2001$

Departamento de Historia del Arte

Universidad de Málaga

Escenografía teatral, festividad y decoración: el dogma de la Inmaculada y su proclamación en la Roma de Pio IX

Los hermanos Díaz de Escovar, dos malagueños dedicados al impulso cultural a través de sus colecciones

El cambio de gusto en la temática pictórica en el tránsito del siglo $\mathrm{XIX}$ al $\mathrm{XX}$ a través de la prensa

La Fábrica de Tabacos de Málaga.

Estudio histórico-artístico de un símbolo de nuestro pasado industrial

Una figura clave para la ilustración española de principios de siglo XX: Francisco Sancha

Los jardines de

Pedro Luis Alonso de Málaga

Un cuarto de baño privado. Fantasía ecléctica y reclamo publicitario

Modernismo e Historicismo arquitectónico:

Delegación de la Consejería de Educación en Cortijo Fischer (Almería)

375 José Manuel Sanjuán López

El Perchel malagueño en la pintura de López Palomo 


\title{
Los jardines de Pedro Luis Alonso, de Málaga
}

\author{
Francisca Pastor Pérez \\ Macarena Recio Pastor
}

Estos Jardines son el resultado del gesto emprendedor del Alcalde Pedro Luis Alonso Jiménez. Su clara voluntad negociadora, mostrada también por los ciudadanos al vender los terrenos a precios asequibles, dota a Málaga de espacios gratos, bellos y apacibles para la convivencia.

The public gardens studied here were possible thanks to enterprising personality of Pedro Luis Alonso Jiménez, Mayor of Málaga, and generosity of citizens who sold needful grounds for their construction in accesible prices. The results of these operations are beautiful natural spaces so nicely and pleased for human relations.

\section{INTRODUCCIÓN}

ANTECEDENTES: TRÁMITES PARA LA REALIZACIÓN DE LOS JARDINES Según Paéz de la Cadena, el desarrollo de la Jardinería requiere una expansión física sobre el terreno, por lo que no es posible en cualesquiera lugar y circunstancia, además de conllevar cuantiosos gastos en materiales, mano de obra y tareas de mantenimiento y por supuesto depende directamente del clima, por lo que las diferencias climáticas marcan los procesos jardineros de las diversas áreas geográficas ${ }^{1}$. Hay que tener en cuenta estas premisas a la hora de examinar las gestiones que a continuación exponemos.

El día 7 de mayo de 1.940, el Excmo. Ayuntamiento pleno se reunió en sesión extraordinaria y el Alcalde dio cuenta de las gestiones llevadas a cabo por el gestor Pedro Alarcón Bryan acerca de los propietarios de los solares enclavados entre la Avenida de Cervantes y las calles Guillén Sotelo y Roma, proponiendo que se hiciera constar en acta, el agradecimiento de la Corporación hacia los propietarios que habían ofrecido la venta de solares y comprar a los Sres.Antonio Álvarez Net, Trinidad Gómez Supervielle, Trinidad Gómez Baratán, Micaela Facia de la Plana y José Álvarez Net los solares que poseían de $1.265 \mathrm{~m}^{2}$ cada uno a razón de 125 pesetas por unidad superficial².

PASTOR PÉREZ, Francisca y RECIO PASTOR, Macarena: «Los jardines de Pedro Luis Alonso, de Málaga», en Boletín de Arte nº 22, Universidad de Málaga, 2001, págs. 333-344. 


\begin{tabular}{|l}
\hline 1. Proyecto inicial \\
de los Jardines \\
de Pedro Luis \\
Alonso por \\
Fernando \\
Guerrero \\
Strachan (1940)
\end{tabular}

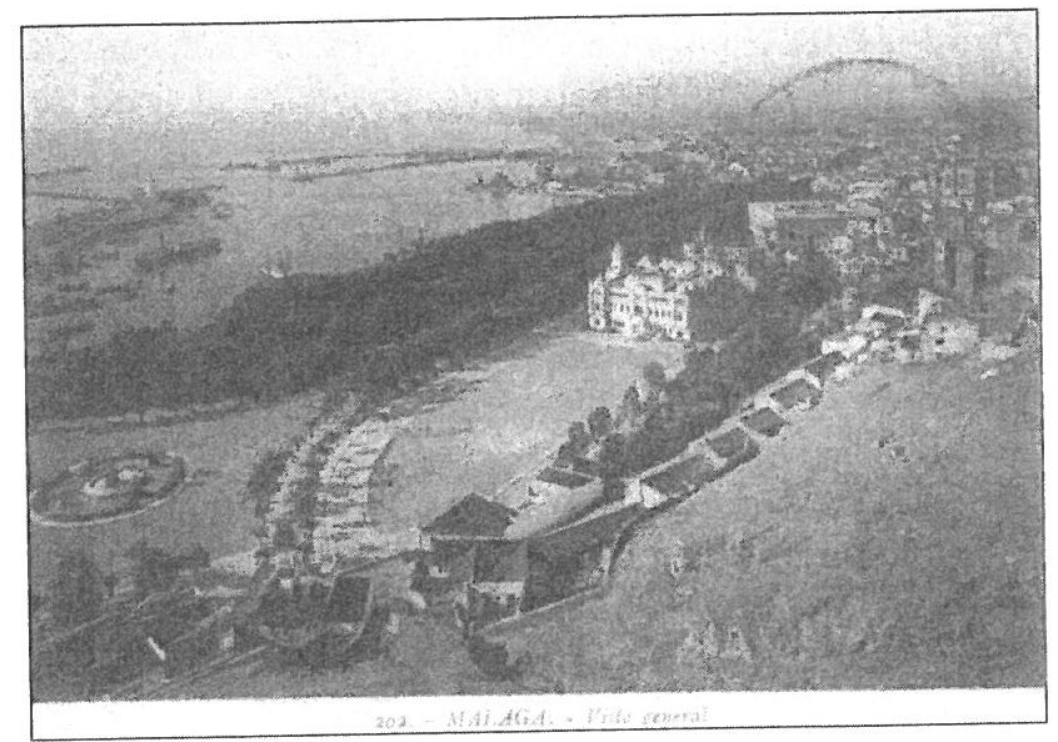

Tras exponer las condiciones de la compra, hizo constar el Alcalde, el día 5 de junio, que no habiendo intervenido en las indicadas negociaciones de compra el propietario José Álvarez Net, los términos y condiciones de adquisición del solar de este señor habrían de ser objeto de acuerdo separado. El Ayuntamiento acordó la propuesta del Alcalde y autorizarle con amplias facultades a resolver las incidencias que pudieran surgir hasta llegar a otorgar la correspondiente escritura pública de compra-venta. Anunció también el Alcalde que comenzarían a construirse los jardines en el solar contiguo al Ayuntamiento, en cuanto entregase el proyecto el arquitecto Académico de Bellas Artes Fernando Guerrero Strachan y Rosado y estuvieran resueltas las dificultades con el último propietario ${ }^{3}$.

Dicho arquitecto presentó, el día 20 de agosto de 1940, al Ayuntamiento de Málaga el proyecto de los jardines que le fueron encargados por el Alcalde Pedro Luis Alonso en el terreno baldío (Fig.1) contiguo al Palacio Municipal. Los jardines fueron proyectados algo más elevados que la rasante de la calle, con lo que se conseguía mayor movimiento y aislamiento, pero se encarecía el presupuesto con las elevadas obras de fábrica, lo cual se advirtió en el examen del anteproyecto; en consecuencia, tuvieron que ser rectificados los planos y reducido su presupuesto. En el nuevo proyecto el arquitecto tuvo que ceñirse al terreno lo más posible, por lo que la monotonía de la planicie del terreno fue disimulada con la variada disposición de las plantaciones; en cuanto al aislamiento, en armonía con su composición de trazado hispano-árabe, fue enmarcado en un seto de cipreses piramidales recortados, formando abierta arquería. Como puede observarse del examen del presupuesto, el importe total de todo lo proyectado se elevaba a la cantidad de

${ }^{1}$ PÁEZ de la CADENA, Francisco: Historia de los estilos en jardinería, Madrid, Ed.Istmo, 1998.

${ }^{2}$ Archivo Municipal de Málaga (A.M.M.) Actas Capitulares (A.C.) 346, fols. 245v y 246 (7-V-1940)

${ }^{3}$ A.M.M.: AC. 346, fols. 251v, 252, 252v (5-VI-1940) 
115.964 '17 pesetas, si bien pudo ejecutarse sin descomponer el conjunto del trazado con un presupuesto reducido de 80.439 '94 pesetas ${ }^{4}$.

D. Fernando Guerrero, al dar las gracias al Ayuntamiento por su encargo y testimoniarle su gratitud, le recuerda las palabras de Francis Bacon en su ensayo Jardines: Dios Todopoderoso plantó en el principio un jardín, lo que es el más puro de los placeres humanos y el mayor recreo para el pensamiento del hombre, sin el cual casas y palacios no son mas que toscas obras de su mano. Los hombres, mientras que las civilizaciones tienden hacia un mayor refinamiento y elegancia, conocen primero la arquitectura monumental, antes de alcanzar la belleza del jardín, como si el arte de éstos no fuera una perfección mayor ${ }^{5}$.

El Excelentísimo Ayuntamiento pleno se reunió en sesión extraordinaria el día 2 de septiembre y dio cuenta del proyecto de construcción de los jardines, decidiendo pasar el proyecto a la Comisión de Hacienda, para que con el gestor delegado de parques y jardines estudiase la forma de llevarlo a cabo y la partida presupuestaria que debía imputarse al gasto, teniendo en cuenta la índole del proyecto y la finalidad que se perseguía ${ }^{6}$, a lo cual la Comisión de Hacienda respondió el 20 de septiembre proponiendo que se autorizara el pago de dichas obras y toda clase de gastos, hasta un total de 81.001 pesetas, con cargo al artículo $3^{\circ}$ del Capítulo XI de la Sección de Gastos del Presupuesto Extraordinario de 1938. Ese mismo día el Alcalde Pedro Luis Alonso decretó pasar el expediente de construcción de los jardines a los Sres: arquitecto municipal, secretario e interventor de fondos municipales, para que informasen si procedía tramitar el expediente sumario de urgencia para eximir las obras de las formalidades de subasta y ejecutarlas por administración directa, a lo que los citados señores responden informando del carácter urgente de la realización de los jardines porque, para convertir el solar en jardín había que aprovechar el otoño y principios de invierno para la plantación de los árboles y sembrar las plantas, puesto que si no, habría que perder un año ${ }^{7}$. El secretario se dirigió por escrito el mismo día al Alcalde para informarle que, debido a que, hasta hacía pocos días, no se había otorgado la escritura de compra-venta de cuatro de los cinco solares en que estaba dividido todo el solar y estando por terminar las gestiones para la adquisición del quinto de los expresados, estimase proceder a la declaración de urgencia de las obras de construcción del jardín exceptuándose así la ejecución de la obra de las formalidades de subasta y efectuarlas por administración o contrato directo. El Alcalde dio cuenta de una propuesta que le había formulado verbalmente el abogado de D. José Álvarez Net, autorizado por éste, para vender al Ayuntamiento el solar que poseía entre la Avenida de Cervantes y las calles Guillén

\footnotetext{
${ }^{4}$ A.M.M. Presupuesto, Legajo 356.

${ }^{5}$ A.M.M.: Expediente, Legajo 356.

${ }^{6}$ A.M.M: A.C 346, fol 269(2-9-1940)

7 A.M.M: A.C. 346 ,fol.271 (21-IX-1940)
} 
Sotelo y Roma, al precio de 125 pesetas el $\mathrm{m}^{2}$, pagadas en veinte anualidades, sin interés, acordando el pleno dejar sobre la mesa este asunto ${ }^{8}$.

El día 21 de Septiembre, en sesión ordinaria el Ayuntamiento acordó: comprar a D. José Álvarez Net el solar que le faltaba, pagado a razón de 7.906'25 pesetas cada una de las veinte anualidades, y autorizar al alcalde para otorgamiento de la correspondiente escritura. Se dio cuenta del proyecto de construcción de los jardines al que se acompañaban dos presupuestos: uno de $115.964^{\prime} 17$ pesetas y otro reducido de $80.439^{\prime} 94$ pesetas También se dio cuenta de un informe de la Comisión de Hacienda en el que propone que se autorice el pago de la construcción de los expresados jardines y toda clase de gastos hasta un total de 81.000 pesetas con cargo al art 3ํ del Cap XI del presupuesto extraordinario de 1.938. Asimismo, se dio cuenta del expediente sumario de urgencia tramitado para eximir de las formalidades de subastas de obra en el que aparecen los informes de los Sres. Arquitecto, Interventor de Fondos y Secretario de la Corporación, en todos los cuales se propugna la declaración de la urgencia de las obras.

El Ayuntamiento acordó: aprobar el proyecto de construcción formulado por el arquitecto D. Fernando Guerrero Strachan y Rosado, con un presupuesto reducido de 80.439'94 pesetas, aprobar el expediente sumario de urgencia y eximir a la obra de las formalidades de subasta. Y, por último decretó encargarle la dirección de las mismas y ejecutarlo por administración directa?

El diario SUR, en su edición del día 2 de octubre de 1940, anunciaba que habían empezado las obras en las que se invertirian de 60 a 70.000 pesetas y que, aunque el proyecto ascendía a 200.00 pesetas, en este año sólo se invertirán las citadas 60 o 70.000, sin perjuicio que el año que viene terminasen las obras proyectadas. Al día siguiente, 3 de octubre, dijo el Alcalde que en poco tiempo el solar quedaría convertido en un maravilloso rincón que honraría a nuestra ciudad, el 10 de noviembre apareció en este diario una foto con los trabajos preliminares de la obra Siguiendo a este diario, el 3 de julio de 1943, en su página $3^{a}$, nos dice que aún no están terminados los Jardines, pues el proyecto de Guerrero-Strachan y Rosado suponía que llevarían cancelas de hierro en los actuales accesos y se esperaba el mayor desarrollo de los cupresos que lo bordean.

\section{LOS JARDINES}

Los Jardines de Pedro Luis Alonso pueden considerarse como antecedentes de la corriente, tan en boga a partir de los años setenta, de concebir el «jardín» como ámbito, como jardín histórico relacionado con la arquitectura a la que siempre está

\footnotetext{
${ }^{8}$ A.M.M.: A.C. 346, fol. 267 (2-IX-1940)

${ }^{9}$ A.M.M.: A. C. 346. fols. 270, 271, 271v. (21-IX-1940)
} 


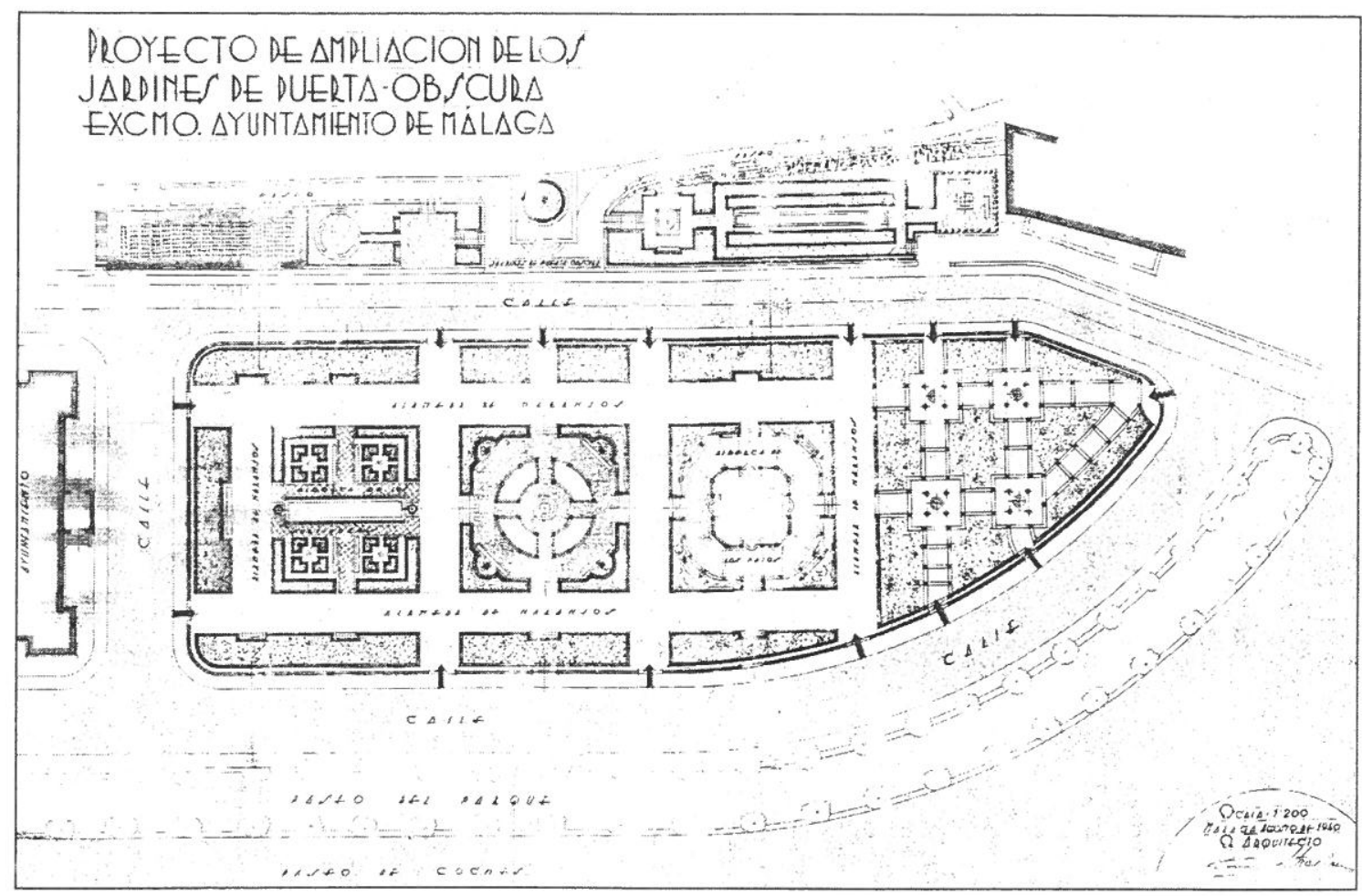

2. Proyecto de ampliación de los Jardines de Puerta Oscura con los de Pedro Luis Alonso

subordinado, protegido y tutelado por los Organismos Públicos. Son cómodos, apacibles para estar a la sombra, pasear, leer y contemplar el bello entorno de la Alcazaba, Jardines de Puerta Oscura, Gibralfaro, que le sirven de fondo, en concordancia con el concepto en materia de urbanismo de jardín público decimonónico ${ }^{10}$. Están cerrados al exterior por setos de cipreses (ya tenemos aquí el primer elemento característico de la jardinería hispano-árabe: su cerramiento) y, siguiendo la tradición árabe, con los elementos que se citan en el Corán: vegetación (productora de placer puro: olores, colores), sombra, árboles frutales (naranjos, casi siempre presente en los jardines hispanos-árabes) y como elemento fundamental: el agua.

\section{MEMORIA}

En el trazado de los jardines se procuró conservar el carácter y sabor del jardín español meridional en terreno llano. Lo extenso del solar y su acentuada horizontalidad hicieron que se subdividiera en varios recuadros a modo de patios y se acentuara la elevación o depresión de su centro con obras de fábrica y la

${ }^{10}$ CAMACHO MARTíneZ, R: (dir.) Guía Histórico Artística de Málaga, Málaga, Arguval, 1992. 


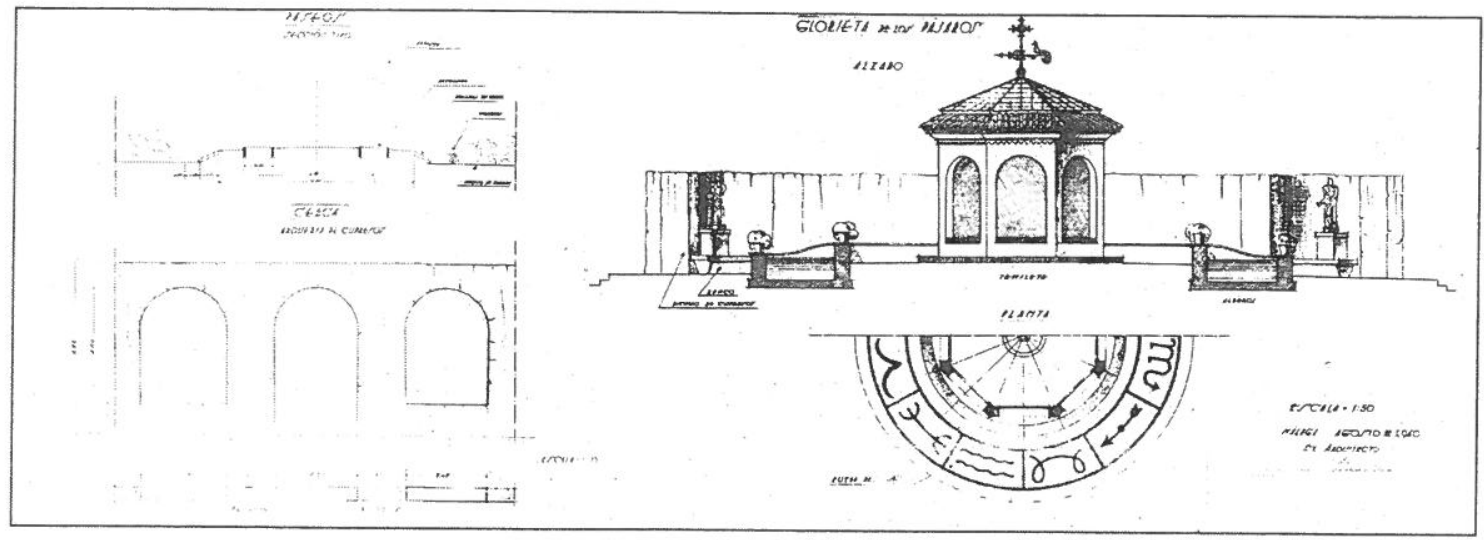

3. Proyecto de resolución del segundo patio de los Jardines de Pedro Luis Alonso con el templete-pajarera

adecuada disposición de las plantaciones. Al estar más elevado que la calle, le permite mayor aislamiento, limpieza y conservación; asimismo las plantaciones y obras de fábrica están dispuestas de forma que revaloricen las perspectivas de los Jardines de Puerta Oscura, la Alcazaba y la Coracha; por eso el arbolado se redujo todo lo posible y se dispuso en grupos concentrados y aislados. Las divisiones del solar en sentido longitudinal y transversal se establecen mediante alamedas de naranjos de $5 \mathrm{~m}$ de ancho las longitudinales y $6 \mathrm{~m}$ las transversales; entre éstas y las calles colindantes quedan amplios rectángulos más bajos que los paseos, sembrados de flores y recercados de molduras recortadas. En estos paseos, de trecho en trecho, se disponían poyetes de ladrillos encalados coronados por grueso tablero de piedra artificial, que servirían de bancos ${ }^{11}$, pero que, al reducirse el presupuesto, fueron cambiados por bancos de madera para el descanso.

\section{LOS JARDINES EN LA ACTUALIDAD}

En el centro del solar se trazaron tres amplios cuadros o patios (FIG.2).

En el primero, hay un estanquillo de poco fondo, estrecho y largo, bordeado de surtidores que se entrecruzan con reminiscencias granadinas ${ }^{12}$. Los paseos van solados de ladrillos y en los ángulos se disponen cuadros de plantas, ajustados a un trazado geométrico en torno a un surtidor, con bancos de ladrillos para el descanso a la sombra de los naranjos.

El segundo patio (FIG.3), ligeramente elevado, se articula en torno a un templete, que, además de servir como elemento centralizador, está destinado a pajarera. De

${ }^{11}$ A.M.M.: Memoria del autor del proyecto. Legajo 356.

12 CAMACHO MARTíNEZ, R.:(dir) Guía histórico artística de Málaga. Málaga, Arguval, 1992. 


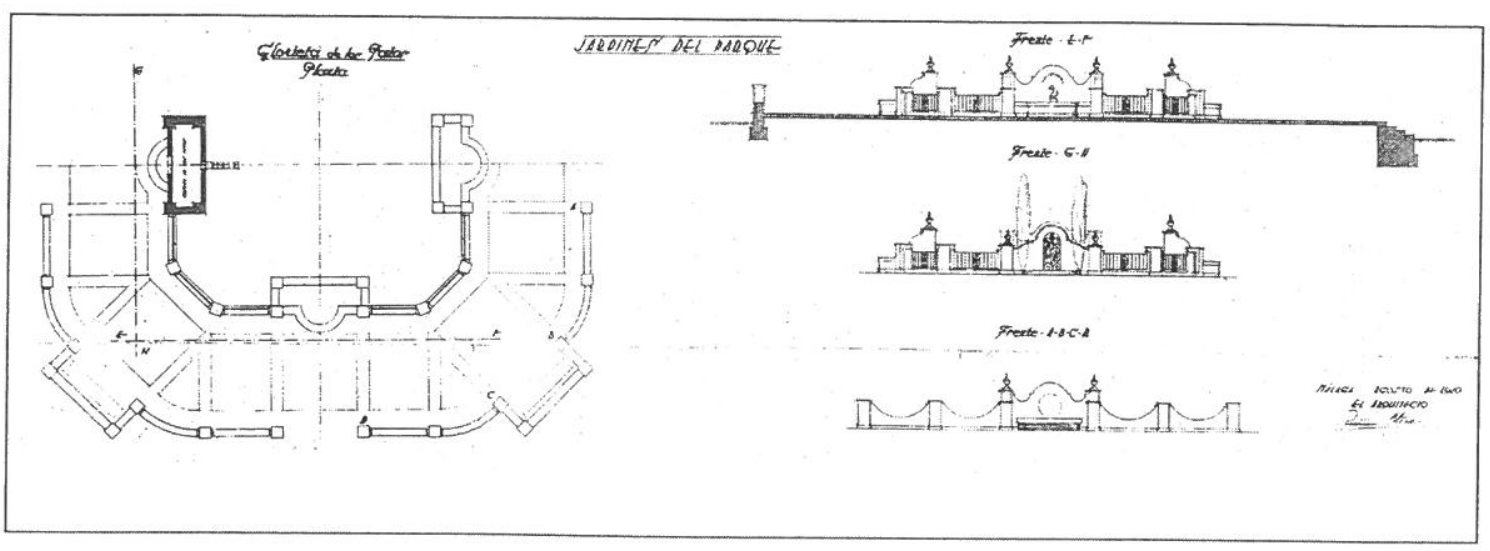

4. Proyecto de resolución del tercer patio de los Jardines de Pedro Luis Alonso con el frente arquitectónico del estanque

trazado octogonal y de obra encalada con cornisa y alero de color gris hoy, antes ocre, está cubierta por tejas de cerámica vidriada de dos colores en su parte inferior y en la superior una claraboya de vidrio da luz a los pájaros, rematada por una esbelta veleta de hierro forjado. Una malla doble protege a los pájaros de las personas que van a visitarlos, aunque dificulta su visión y hace que no se puedan ver bien. El suelo que circunda a la pajarera es un círculo de trozos de mármol recortado, que - al bajar un escalón de ladrillo- nos lleva al precioso enchinado bicolor con los signos del Zodiaco. A su alrededor se disponen en forma circular cuatro pequeñas albercas (que en la actualidad han sustituido su función de contención de agua por arriates de vegetación) con poyete de fábrica, de poca elevación, para colocar macetas. Se enmarca el patio por cipreses recortados, que forman en los ángulos unas exedras proyectadas para que sirvieran de fondo a cuatro esculturas colocadas sobre pedestales de fábrica de ladrillos encalados y que, con los recortes presupuestarios, quedaron para acoger las farolas que iluminan la glorieta.

En el tercer patio (FIG.4) hay un amplio estanque destinado a cisnes y patos. Esta alberca de movida planta presenta en sus lados tres pilones, de fábrica de ladrillo, de silueta festoneada de paños lisos y encalados, que fueron proyectados con remates y mascarones de cerámica muy al gusto del siglo XVIII, pero por las razones presupuestarias que condicionaron el proyecto, no llegaron a colocarse. En los ángulos se coloca un barandal de hierro forjado. En el cuarto lado de la alberca, a juego con los pilones, se dispuso la casita que sirve de albergue a los patos. Rodea el recinto una obra igualmente festoneada y encalada, que incluye cuatro bancos cuyos respaldos están abiertos con grandes óculos y rematados los pilares por cerámica popular hoy desaparecida. El suelo es de ladrillo alrededor de la alberca y, a

\footnotetext{
${ }^{13}$ Según nos informan los cuidadores del jardín, el mármol procedía de lápidas funerarias, lo cual se puede constatar en las inscripciones que aparecen en algunos de los fragmentos
} 
continuación, de mármol recortado de varios colores ${ }^{13}$. En uno de los pilones hay una placa conmemorativa de mármol fechada en 1.948 y en la que dice que el Alcalde Pedro Luis Alonso consiguió para Málaga la bella realidad de estos jardines. Esta placa fue colocada por decisión del Ayuntamiento, a propuesta del Alcalde Estrada Segalerva para honrar la memoria del ex Alcalde que había hecho tantas obras y tan buena labor en beneficio de Málaga

En el extremo NE del jardín, figuras poligonales formadas por setos de cipreses recortados dan cabida a variada vegetación, y entre ella hay cuatro fuentes de ladrillo y silueta festoneada, que acogen a cuatro surtidores.

\section{ANÁLISIS DE LOS PRESUPUESTOS}

En primer lugar, hemos de señalar que el análisis se ha realizado sobre tres presupuestos: los dos ya mencionados, más un tercero con fecha 1 de septiembre de 1.940 de 136.624 '62 pesetas, del que no hemos hablado porque excedía con mucho el presupuesto fijado por el Ayuntamiento, los dos primeros consistían en un presupuesto como tal y las reducciones que se le hicieron para ajustarse a la cantidad 81.000 pesetas admitidas por la Corporación Municipal. Sin embargo, fue el proyecto del tercer presupuesto el que se ejecutó; si bien seguía con bastante aproximación al primero, vemos cómo se elevaba su cuantía en los siguientes conceptos.

En el vaciado de tierras y terraplenado observamos que frente a las $3.135^{\prime 2} 21$ ptas. del primer presupuesto el ejecutado se elevaba a 8.496`59 ptas.

Las cimentaciones que suponían $6.356^{\prime} 16$ ptas. con las reducciones que se le realizaron en el actual presupuesto supusieron $30.453^{\prime} 48$ ptas.

Las obras de fontanería, alumbrado y alcantarillado que suponían en el primer presupuesto $11.387^{\prime} 50$ ptas., en el nuevo proyecto supusieron 13.500 ptas.

Además, observamos que los elementos que a continuación se enumeran no llegaron a materializarse:

- muros de mampostería careada ................. 8.729'43 ptas.

— muros y muretes de mampostería ordinaria . . . . . . . 15.543'55 ptas.

— fábrica de ladrillos en bancos ..................1.468' 69 ptas.

- tableros de piedra artificial en bancos .............4.256 ptas.

En el Patio de los Surtidores:

- fuentes surtidores sobre columnas ................600 ptas.

En el Patio de los Pájaros:

- columnas de piedra .........................2.400 ptas. 


Q- arrínilos Los jardines de Pedro Luis Alonso, de Málaga

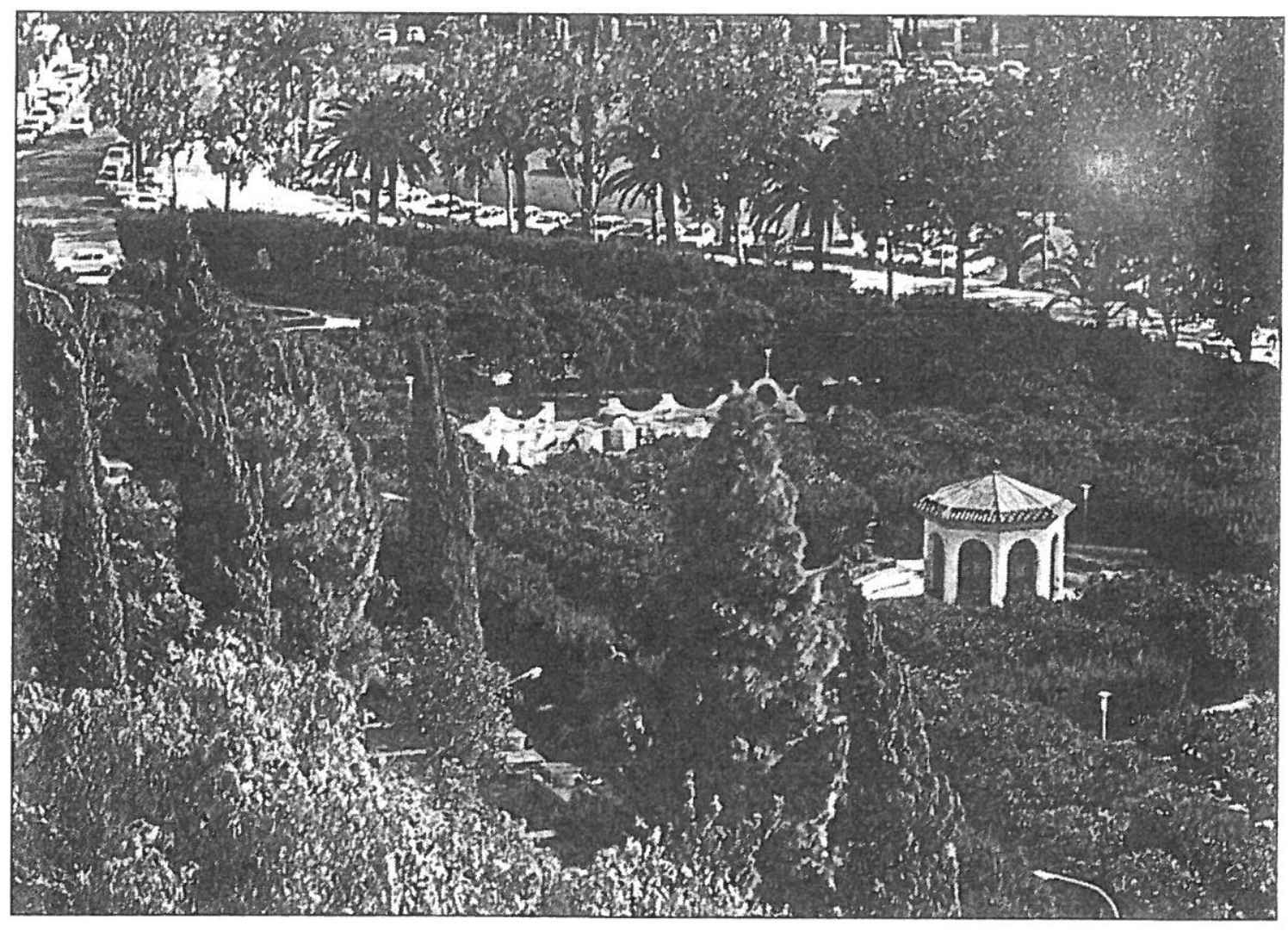

5. Vista de los Jardines de Pedro Luis Alonso desde los Jardines de Puerta Oscura

— fábrica de ladrillo en pedestales para estatuas . . . . . . 1.733'76 ptas.

Glorieta del Estanque de los Patos:

- remates cerámica . . . . . . . . . . . . . . . . . . . . 1.200 ptas.

- pez de cerámica en los pilares . . . . . . . . . . . . . . . 600 ptas.

- mascarones de cerámica ... . . . . . . . . . . . . . 1.200 ptas.

El presupuesto de ejecución material de las obras fue de 136.624'62 ptas. que se elevó a la cantidad aproximada de $175.833^{\prime} 58$ ptas. con los gastos de plantaciones, jardinería y honorarios de proyecto y dirección ${ }^{14}$.

${ }^{14}$ A.M.M.: Memoria, Legajo 356. 


\section{APÉNDICE DOCUMENTAL}

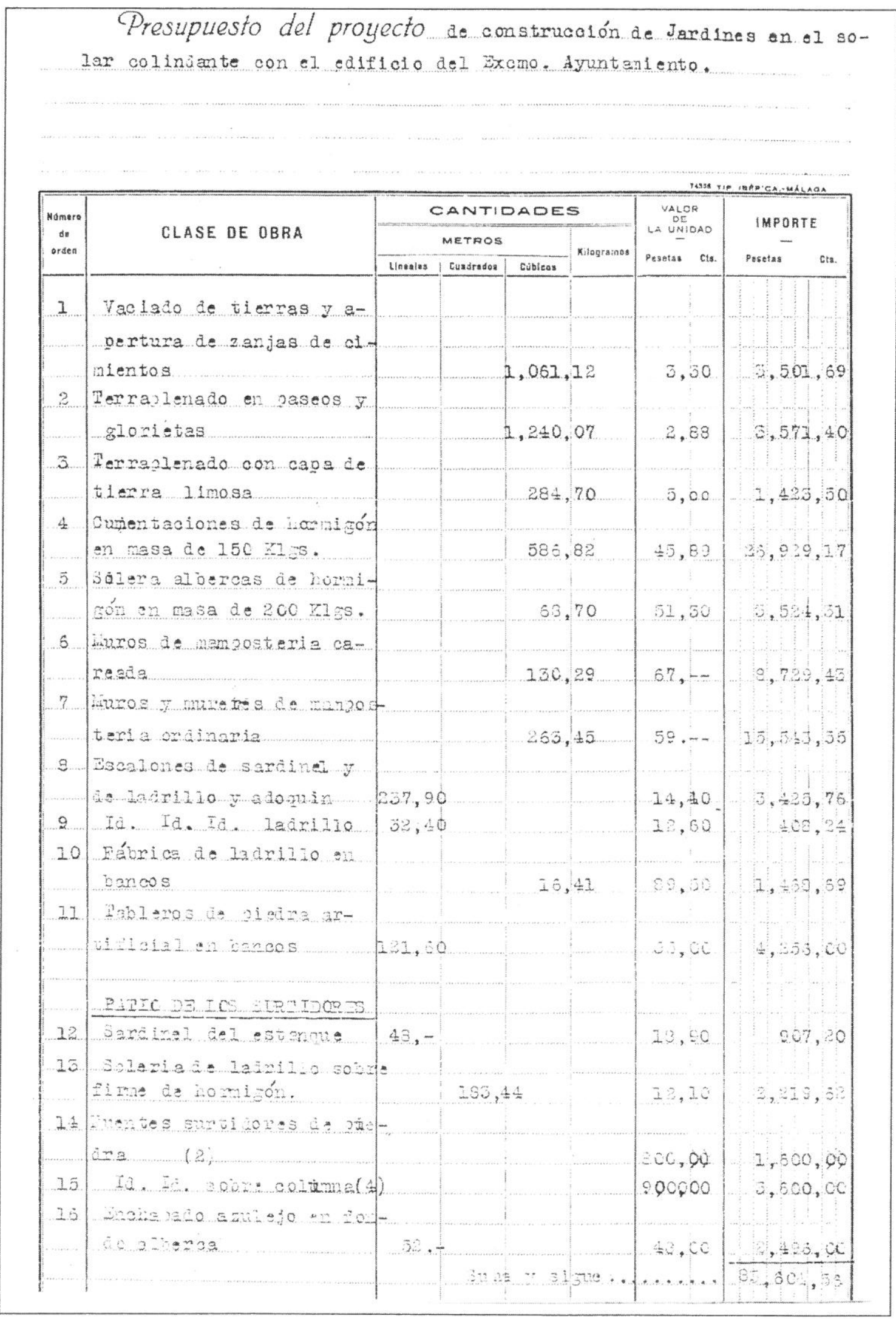




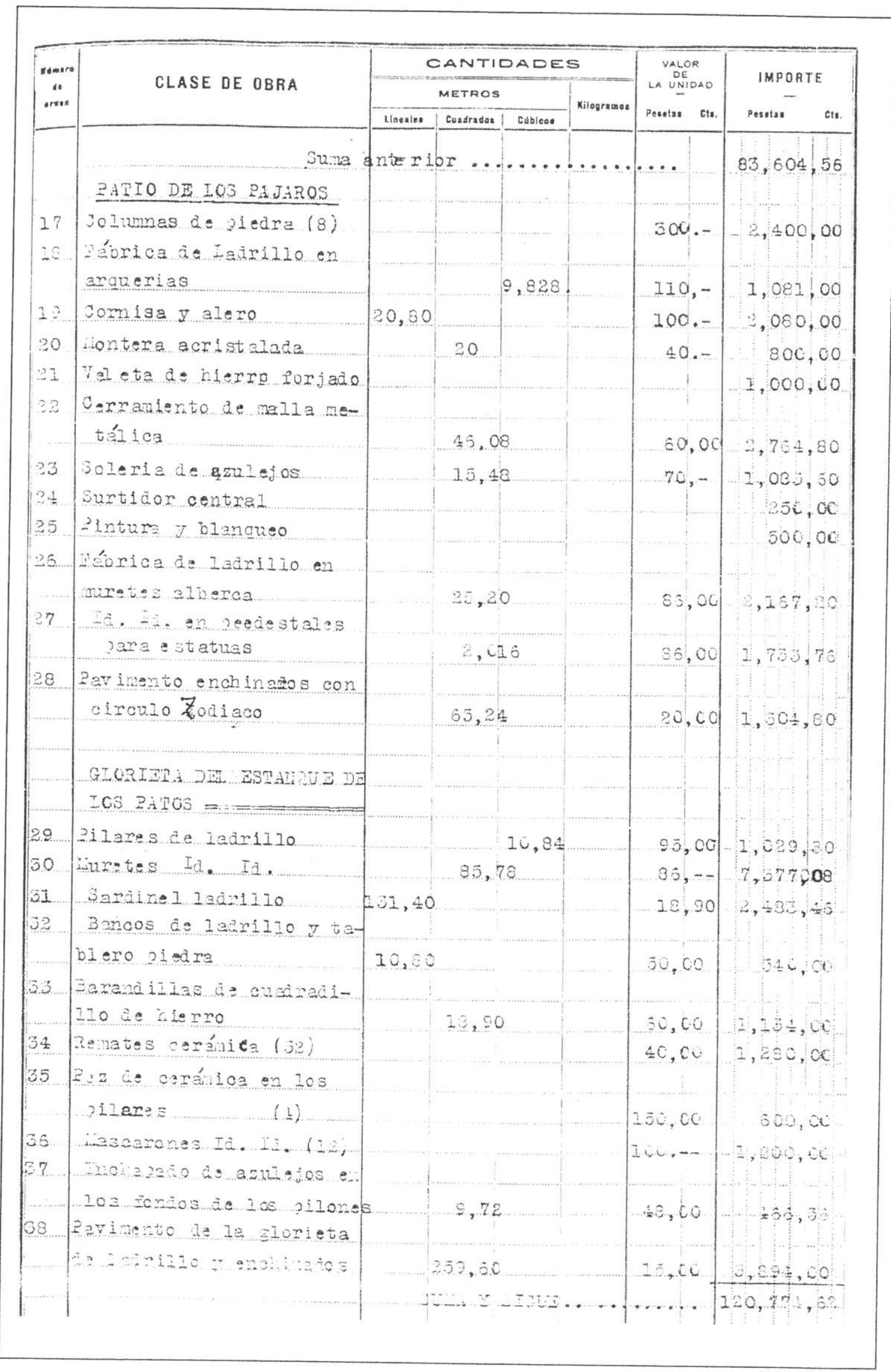




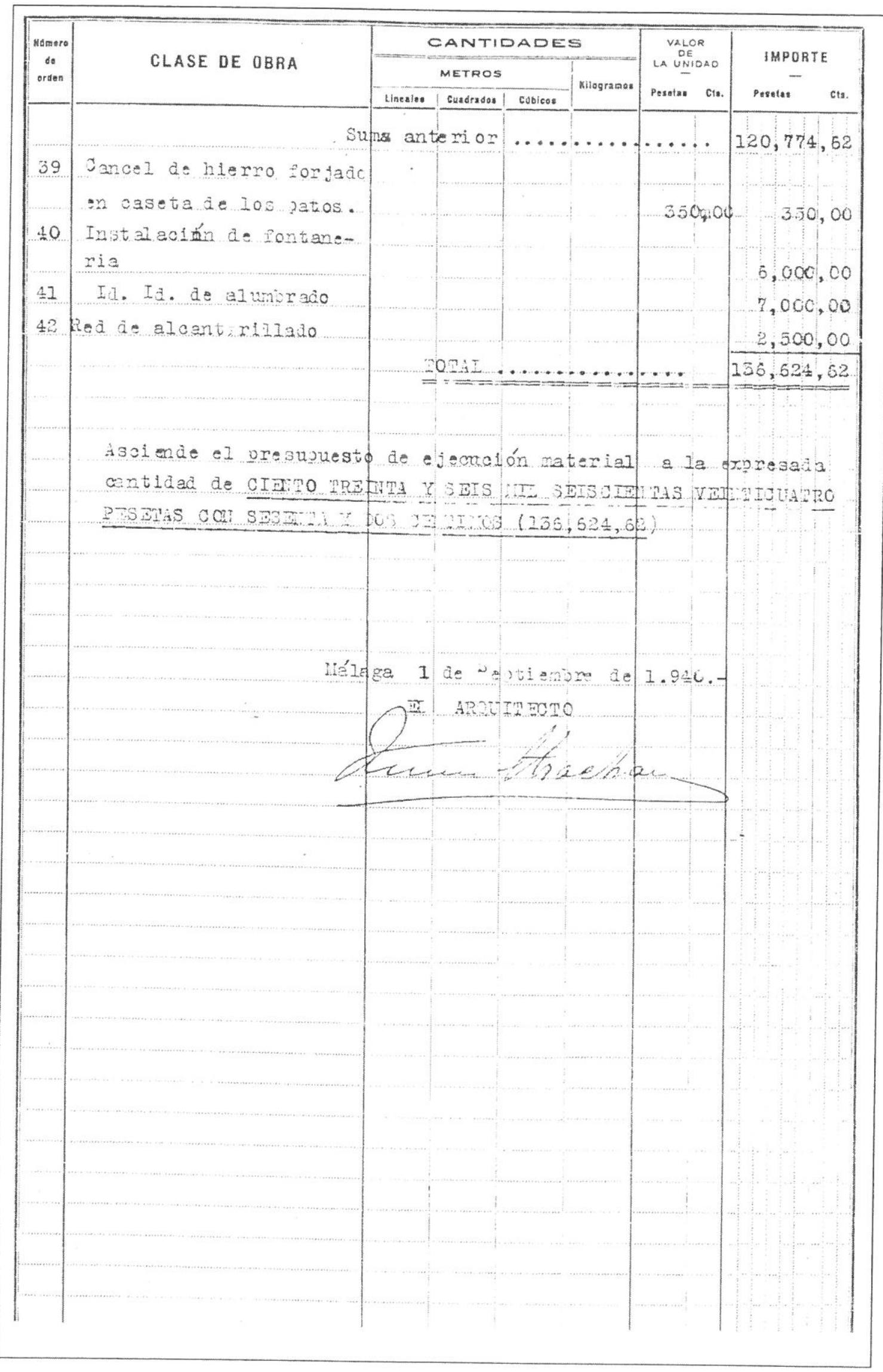

